typischen Hängetälern, prächtig ausgebildete, den Ufern der Russell-Bucht, des Nunadakfjords etc. parallele Erosionsterrassen und begründet ausführlich seine Anschauung, dass die Hängetäler und die Übertiefung der Haupttäler nur durch eine kräftig erodierende Wirkung der Gletscher entstanden sein können. $\mathrm{Ob}$ auch in diesem Gebiete mehrere Taltröge ineinandergeschachtelt sind, lässt sich aus den Bildern nicht sicher erkennen; verschiedene Spuren höher gelegener Terrassenabsätze lassen darauf schliessen, dass dem so ist; doch fehlen bis jetzt Karten grösseren Massstabes, welche diesen Schluss stützen könnten. Um die erodierende Wirkung des Eises zu verstehen, muss man, wie TARR hervorhebt, für den ganzen Prozess einen genügend langen Zeitraum ansetzen und beachten, dass mächtige, stark vorschreitende Gletscher ihren Untergrund ungleich stärker bearbeiten, als die ruhig fliessenden Eismassen der Gegenwart genau so wie eine Hochflut im Flusstal weit grössere Veränderungen hervorruft, als der normal gehende Fluss.

Einen weiteren Beitrag zur Lehre von der glazialen Exrosion bringt QUENSEL, welcher die Verlegung der Wasserscheide im Gebiete des Lago San Martin in Patagonien von der Achse der Kordillere weg nach Osten hin studiert hat. Er findet, dass die Eismassen der letzten Kordillerenvergletscherung stark erodierten, dass die ostwärts abfliessenden Gletscher grosse Schuttmassen in die präglazialen Flusstäler der Pampas vorschoben und hier den Abfluss der Wassermassen verhinderten. Die glaziale Erosion gab zur Bildung der grossen fjordartigen Seebecken Veranlassung, welche wie der Lago San Martin, Lago Argentino, Ultima Esperanza etc. in ihren Verzweigungen den Fjorden auf der Westseite der Cordilleren entsprechen und mit diesen durch tiefe Einsattelungen der Hauptkette in Verbindung stehen. Durch die Erosionsarbeit der eiszeitlichen Gletschermassen wurde die Wasserscheide um beträchtliche Strecken ostwärts verlegt.

\title{
Fortschritte der geologischen Forschung im Schweizer Juragebirge, insbesondere in dessen nördlicher Hälfte.
}

\author{
Von H. Gerth, Bonn.
}

1. F. Machacek : Der Schweizer Jura, Versuch einer geomorphologischen Monographie. Petermans's Mitteilungen. Ergänzungsheft 150. Gotha 1905. S. 1-143 mit Karte und Profiltafel.

2. A. Buxtorf: Geologische Beschreibung des Weissensteintunnels and seiner Umgebung. Beiträge zur geologischen Karte der Schweiz. 21. Bern 1907.

3. F. MürnberG: Geologische Karte der Lägernkette, 1 : 25000 , mit Erläut. Bern 1902.

Geologische Karte des unteren Aare-, Reuss- und Limattales, 1: 25000 mit Erläut. Bern 1905.

Das Schweizer Juragebirge hat der Juraformation den Namen gegeben, weil ihre Ablagerungen in ihm grosse Verbreitung besitzen. Aber noch eine andere Eigentümlichkeit macht uns das Studinm der Juraschichten in diesen Bergen besonders interessant. Das Jurameer besass im Osten und Westen des heutigen Gebirges verschiedene Tiefenverhältnisse, es wurde von einer verschiedenen Lebe- 
welt bevölkert und in beiden Meeresteilen kamen ganz verschiedenartige Sedimente zum Absatz. So kommt es, dass uns heute die Ablagerangen der Juraformation in zwei verschiedenen Ausbildungen entgegentreten, so dass es grosse Schwierigkeiten verursachte, die richtigen Altersbeziehungen der einzelnen Schichten in den beiden Faziesbezirken klar zu stellen ${ }^{1}$ ). Während sich der Lias, ebenso wie die im östlichen Jura zutage tretende Trias allenthalben eng an die ausseralpine, europäische Entwickelung dieser Schichten anschliesst, macht sich der Faziesunterschied zuerst im Dogger geltend. Entlang dem Südrand des Jura und in seinem ganzen östlichen Teil beobachten wir eine vorwiegend mergelige Ausbildung, wie in Schwaben, während in dem grösseren, die westlichen Partien des Gebirges umfassenden Bezirk eine oolithisch-kalkige Entwickelung der Schichten herrscht, wie wir sie aus Lothringen, überhaupt der ganzen Umrandung des Pariser Beckens bis nach England hinein kennen. Ebenso scharf sind die Gegensätze im Malm. Mächtige Tone mit individuenreicher Cephalopodenfauna, die oben von Tonkalken and Mergeln bedeckt werden, repräsentiexen im westlichen Bezirk das Oxford. Am Süorande des Jura und im östlichen Teil fehlen diese Schichten ganz, oder die leitenden Ammoniten liegen zusammen mit denen tieferer Zonen in einem stellenweise konglomeratischen Eisenoolith, der uns Aufarbeitung and Transgression anzeigt. Wohl war der Südrand des Jura zu dieser Zeit teilweise und vorübergehend Festland, ein Teil der vindelizischen Bodenschwelle. Später sehen wir den westlithen Bezirk wieder von einem flachen Meer bedeckt, in dem mächtige, korallogene Kalke und Oolithe zum Absatz kamen, während sich im Süden und Osten ein tieferes Meer mit Schwammbildungen und einer mehr pelagischen Cephalopodenfauna ausdehnte. Erst nachdem man erkannt hatte, dass die mächtigen Ablagerungen des Oxford am Südrande und im Osten des Jura ganz fehlen, kam man dazu, die Mergel. und Kalke des Argovien des Ostens mit den massigen Korallenkalken des Rauracien im Westen zu parallelisieren. Früher hatte man im Argovien ein Äquivalent des Oxford gesehen, zumal beide Stufen in der Orographie des Gebirges in gleicher Weise hervortreten. Beim Aufbruch der Gewölbe durch die Erosion entstehen in den Mergeln und Tonen des Oxford und Argovien Längstäler, sogen. Komben, über die die Kalke des Ranracien und höheren Malm als Kämme emporragen. Die Grenze zwischen den nunmehr als gleichalterig anzusehenden Ablagerungen des Argovien und Ranracien verläuft von Liestal über Roche bei Moutier, Biaufond nach Salins. Auch im oberen Malm treten die Faziesunterschiede hervor, nur reichen die rein kalkigen, oolithischen Flachseebildungen noch etwas weiter nach Süden bis zu der Linie Schwarzwald, Önsingen, Bellegarde, Bas-Bugey. Gegen Ende des Malm führte eine Heraushebung im Norden des heutigen Gebirges zur Trockenlegung der nördlichen Partien. Durch Austernbänke, die sich von Belfort über Delsberg, Solothurn, bis in den schwäbischen Jura verfolgen lassen, wird der ungefähre Verlauf der Küstenlinie angedeutet. Die Trockenlegung schritt weiter nach Süden vor und am Schlusse der Juraepoche sehen wir fast das ganze heutige Gebirge einen Teil des mitteleuro-

1) Für den weissen Jura ist die Parallelisiernng der Schichten besonders durch die Untersuchangen RoLLiens geklärt worden; dieser Jurageologe hat die Ergebnisse der stratigraphischen Forschung der letzten Jahre kürzlich in einem Vortrage auf dem Kongress der Association Franc-Comtoise (Pontarlier 1909) zusammengefasst. 
päischen Festlandes bilden, für das die Ubereinstimmung brackischer PurbeckAblagerungen der Gegend von Neuchâtel mit denen Norddeutschlands bezeichnend ist. Der Innenrand und die Südgrenze des eigentlichen Jura zwischen Biel, Morteau, Pontarlier und Bellegarde bildete die Küste des damaligen Meeres. Aber schon bald rückte die Flut wieder vor und immer weiter sehen wir die Stafen der unteren Kreide nach Norden übergreifen. Ablagerungen der oberen Kreide sind nur in isolierten Fetzen im Süden erhalten. Sie ermöglichen es uns nicht, die Ausdehnung des Meeres der damaligen Zeit, die offenbar wiederholten Schwankungen unterworfen war, mit Sicherheit festzustellen. Die nördlichen Teile des Jura dürfte das Kreidemeer nie mehr erreicht haben. Hier war Festland, das sich zn Beginn des Tertiär halbinselartig wieder weit nach Süden erstreckte. Die als Verwitterungsprodukte anzusehenden Bohnerzbildungen und Süsswasserkalke charakterisieren dieses Land. Marine Ablagerungen des Eozäns fehlen im ganzen Jura, erst im Oligozän kehrt das Meer zurück. In wechselnden Transgressionen und Regressionen greift es bald von Norden, bald von Süden äber die Jurahalbinsel über. Vorübergehend kommt es auch zu einer Verbindung des nördlichen und südlichen Oligozänmeeres darch eine den Jura quer durchziehende Senke, die die südliche Fortsetzung des Rheintalgrabens bildet. Im Miozän ist der Südrand des Gebirges von dem europäischen Mittelmeer bedeckt, während der Norden sich zu heben beginnt. Zur Zeit der grössten Ausdehnung verläuft die Uferlinie über Chaux de Fonds, Delsberg, Liestal bis zum Randen. Diese parallel laufenden, das Gebirge in südwestnordöstlicher Richtang durchziehenden Linien saben wir schon in der Jurazeit eine grosse Rolle in den Tiefenverhältnissen des Meeres spielen. Am Schlusse des Miozäns zieht sich das Meer zurück, und allenthalben kommen in isolierten Becken Süsswasserkalke zum Absatz. Jüngere Ablagerungen des Tertiärs kennen wir aus dem Jura nicht, erst wieder die Bildungen der Diluvialzeit. Inzwischen sind aber die eben geschilderten, bis dahin flachliegenden Sedimente zu einem Gebirge aufgefaltet worden.

Der Zeitpunkt der Faltung ist also ziemlich genau fixiert; schwieriger ist es schon, sich ein Bild von dem faltenden Vorgang im einzelnen zu rekonstruieren. Wir müssen zu diesem Zwecke von den Erscheinungsformen atısgehen, in denen das Gebirge uns heute entgegentritt. In tektonischer und morphologischer Hinsicht lassen sich drei Formen unterscheiden: Kettenjura, Plateaujura und Tafeljura. Der Kettenjura besteht, wie sein Name besagt, aus Antiklinalen, die durch sich lang hinziehende Synklinaltäler voneinander getrennt werden. Die Talbildung schliesst sich dem ursprünglichen Faltenwurf eng an, Täler im Scheitel der Antiklinale sind selten oder spielen eine untergeordnete Rolle. Die herrschende Gewölbeform ist eine Antiklinale mit'steilen Schenkeln und flachem Scheitel. Nicht selten stellen sich auf dem Nordschenkel Störungen ein, die die Falten nach Norden übergelegt erscheinen Iassen. Für die Ketten am Innenrande des Gebirges ist ein derartiger Bau besonders charakteristisch. Dem südlichen Kettenjura ist im Nordwesten der Plateaujura vorgelagert. Die Scheitel der Antiklinalen und die Böden der Synklinalen werden weiter und flacher, die orographische Gliederung in einzelne Ketten schwindet, die Landschaft nimmt plateauartigen Charakter an. Im äussersten Westen, am Doubs, wird der Plateaujura noch einmal durch eine Zone stärkerer Auffaltung und Zusammenschubs begrenzt. Der nördliche Teil des Kettenjuras zeigt einen an- 
deren Charakter als der südliche. Die Faltung scheint eine intensivere gewesen zu sein, die Antiklinalen wurden nach der Faltung hauptsächlich durch Zusammenbruch stärker gestört. Die Kotten sind heute tiefer aufgebrochen und werden häufiger von Quertälern, Klusen, durchschnitten. Wie wir wissen, hat die Faltung hier durch die im Norden liegenden Massive des Schwarzwaldes und der Vogesen eine Stauung erfahren. Zwischen dem Fuss dieser alten Massen und die sich zusammendrängenden Falten wurde eine Juraplatte eingeklemmt, die von der faltenden Bewegung nicht ergriffen worden ist. In der Verlängerung des zwischen den beiden alten Halbhorsten eingebrochenen Rheintalgrabens drangen die Falten weiter nach Norden vor, wihhrend im Innern des Gebirges, im Bereich der schon erwähnten Senke, weite, von mächtigen Tertiärschichten erfüllte Becken unaufgefaltet blieben. Der Tafeljura erleidet hierdurch eine Unterbrechung. Im Osten, südlich des Schwarzwaldes, ist die Zahl der Ketten sehr reduziert. Die nördlichste dringt an einer Flexur, die bei der Aaffaltung zur Überschiebung wurde, in mehreren Schuppen über die ihr vorgelagerte Juratafel nach Norden vor.

Erörtern wir nach diesem kurzen Ausblick auf Bau- und Oberflächengestalt des Gebirges die über seine Auffaltung bestehenden Hypothesen. Brückner nimmt eine zweifache Dislokationsperiode an, zwischen beiden soll das ganze Gebirge zu einer Rumpfläche eingeebnet und dann einer erneuten, sehr jungen Faltung unterworfen worden sein. Wir könneu MACHACEK nur recht geben, wenn er die von BrücrNer angegebenen Beweise nicht für zwingend hält; ausser der Hauptfaltung beschränkten sich weitere Dislokationen auf Heraushebung und Absenkung einzelner Teile. Aber auch die Rumpffäche scheint nur für einen kleinen Teil des Gebirges bestanden zu haben. Nur an wenigen Stellen in der Umgebung des Plateaujura treten uns heute zu einer annähernd ebenen Oberfläche abgetragene Falten entgegen, während der Plateaucharakter des Plateaujura in erster Linie durch den tektonischen Bau bedingt ist. Der Kettenjura aber mit seinen hoch aufragenden, geschlossenen oder tief aufgebrochenen Gewölben dürfte wohl niemals zu einer Rumpffäche eingeebnet gewesen sein. Vor allem vermissen wir heute jede Spur der pliozänen Geröll- und Trümmermassen, durch die die Synklinaltäler des Kettenjura bei der Einebnung bätten ausgefüllt werden müssen. Machaokr nimmt an, dass die Auffaltung des Gebirges im äussersten Nordwesten mit der westlichen Randzone des Plateaujura begonnen hat und von dort nach Südosten fortgeschritten ist. Die innersten Ketten des Jurabogens sind also die jüngsten. Er stützt sich bei dieser Annahme darauf, dass die Falten der westlichen Randkette heute die stärkste Abtragung erfahren haben und auch im Plateaujura das Alter der gebirgsbildenden Schichten in der Regel nicht nur von Norden nach Süden, sondern auch von Westen nach Osten abnimmt. Die nach Süden zunehmende Höhe des Gebirges soll vicht eine Folge stärkerer Aufwölbung, sondern der verschieden langen Dauer der erodierenden und denundierenden Kräfte sein. Auch BvхтовF nimmt im nördlichen Jura ein Fortschreiten in der Bildung der Ketten von Nordwesten nach Südosten an. Diesem Forscher verdanken wir auch eine neue Theorie über den Faltungsprozess, die uns viele Eigentümlichkeiten des .Turagebirges erklärt und für die Entstehung der Faltengebirge überhaupt von weittragender Bedeutung sein dürfte ${ }^{1}$ ). Die Tatsache, dass im Kern der Juragewölbe auch dort, wo sie selar tief aufge-

${ }^{1)}$ Zur Tektonik des Kettenjura (Ber. Oberrh. geol. Ver. 30, 1907). 
brochen oder nach Norden auf den Tafeljura überschoben sind, nirgends ältere Schichten als die Anhydritgruppe des Muschelkalkes zutage kommen, führte ihn zu der Annahme, dass bei der Bildung der Falten in den weichen Mergeln der Anhydritgruppe eine Loslösung der höheren Schichten von ihrer Unterlage stattgefunden hat. Sie allein wurden von der Iraltung ergriffen, während das Grundgebirge derselben Widerstand leistete and in Rahe blieb. Die Steilstellung der Schenkel der Antiklinalen, sowie ihre Konvergenz gegen die Tiefe hin, die BшхтокF an einigen Stellen konstatiert haben will, finden so leicht ihre Erklärung. Das Juragebirge verdankt wie die Alpen, von denen es ja nur ein Seitenast ist, einem aus Südosten kommenden Schub seine Entstehung. An seiner heutigen Stelle befanden sich die mesozoischen Sedimente, wie wir gesehen haben, schon vor Beginn der Faltung in gehobener Lage und nur von wenig mächtigen Tertiärablagerungen bedeckt. Im Schweizer Mittelland lagen sie dagegen nnter mächtigen Molasseschichten begraben. So kam es, dass sich dort der die Alpen bildende Tangentialschub noch einmal äussern konnte, indem er die höheren Sedimente von ihrer Unterlage loslöste und faltete, schaf er einen dem Hauptgebirge parallelen Gebirgsbogen.

Bald darauf in der Diluvialzeit erlitt das junge Gebirge eine bedeutende Abtragung. Wie wir schon lange wissen, wurde der Jura von der alpinen Vergletscherung zur Zeit ihrer maximalen Ausdehnung erreicht. Die umfassenden Untersuchungen von PENCK und BrÜCKNER, die in dem Werke "Die Alpen im Eiszeitalter" niedergelegt sind, werfen auch auf die Beziehungen des Juragebirges zu den verschiedenen Vereisungsperioden nenes Licht. Im Innern des Gebirges sind uns nur die Spuren der grössten, der Risseiszeit erhalten. Anzeichen früherer Vergletscherungen sind durch sie verwischt worden. Nach der Ansicht BrüCKNER's stauten sich die Alpengletscher, die sich im Alpenvorlande zu einer einheitlichen Eismasse vereint hatten, an den Juraketten. Durch Lücken and über Einsattelungen in ihnen drang das Eis in das Innere des Gebirges ein und breitéte sich in den Synklinaltälern wieder aus. Das ganze Gebiet wurde mit weither verfrachtetem alpinen Material überstreut, das uns heute ein gutes Hilfsmittel bietet, die damalige Ausdehnung des Eises zu rekonstruieren. Die höchsten Höhen des Jura scheinen die alpinen Gletscher nicht erreicht zu haben. Die Stirnmoränen der Hauptvereisung sind uns im Gebirge nur unzusammenhängend erhalten. Sie dürften sich von Rheinfelden bei Basel über Liestal, die Passwangkette, am Südfuss der Hohen Winde, des Raimeux und Moron entlang laufend, äber Bellelay, Maîche, Le Russey und Morteau zum Mont Chaumont, über Ornans im Louetal und schliesslich nach Westen ausbiegend, bis in die Gegend von Salins hingezogen haben. Im Süden wurde das Gebirge von dem vereinigten Rhône- and Isère-Gletscher vollkommen gequert, und die Eismassen erstreckten sich hier weit nach Westen bis zum Rand des französischen Zentralplateant. Weit geringere Ausdehnung besass die folgende, die Würmeiszeit. Nur ein westlicher Arm des Rhonegletschers überschritt das Gebirge noch ganz im Süden, der Ostarm wurde durch den ihm entgegentretenden Wall nach Nordosten abgelenkt, ohne in die Ketten einzudringen. Nordwärts bis in die Gegend von Solothurn reichend, zieht sich seine mächtige Stirnmoräne am Südfusse des Gebirges hin. Die grösseren Höhen im Süden des Jura waren der Ausgangspunkt einer wohl entwickelten Lokalvergletscherung, die sich auch auf den hochliegenden Plateaujura ausdehnte. Aus dem weniger hohen Norden kennen wir 
sichere Sparen lokaler Vereisungen nicht. Die Erosionswirkung des Eises tritt im Jura nicht so hervor wie in den Alpen. Nur ganz im Süden, im Rhonegebiet, sind uns Kare, übertiefte Haupttäler und trogförmige Talwannen heute noch erhalten. Immerhin dürfte die Diluvialzeit mit ihren bedeutend erhöhten Niederschlagsmengen und wesentlich verstärkten Erosionsperioden ein Hauptfaktor bei der Herausbildung des heutigen Jurareliefs gewesen sein.

Während so allgemeine Fragen der Stratigraphie, des Gebirgsbaues und der Morphologie im Juragebirge beständig ihrer Lösung entgegengehen, schreiten auch Detailuntersuchungen verbunden mit kartographischen Aufnahmen in grossem Massstabe rüstig vorwärts. Die bei einer Durchtunnelung des Weissenstein gewonnenen Resultate veranlassten BuxтoRf, ein Stück der Kette im Massstabe 1: $25000 \mathrm{zu}$ kartieren. Sie besteht in der Umgebung des genannten Berges nicht aus einer einfachen Antiklinale, sondern hat zwei aus Schichten des Dogger gebildete Gewölbekerne. Die Rötifluhfalte mit im Osten steil gestelltem Südschenkel klingt nach Westen hin aus in einer Flexur des Südschenkels. An ihre Stelle tritt die Stahlfluhfalte mit steilem Nordschenkel. Dort, wo die beiden Gewölbekerne nebeneinander bestehen, treten in den benachbarten Schenkeln Brüche und Ausquetschungen auf. Die Kette erleidet an dieser Stelle eine schwache S-förmige Krämmung, da die Hauptfalte von der Südseite auf die Nordseite übergeht. Wie der Tunnel ergeben hat, nehmen die höheren Schichten des Nordschenkels unter Tage Südfallen an, so dass die Mulde von Gänsbrunnen, die die Weissenstein- von der Farisbergkette trennt, nach Norden überkippt ist. Der Bau der Kette wird uns durch eine Serie von Profilen, die in gleichen Abständen durch das untersuchte Gebiet gelegt sind, vorzüglich veranschaulicht.

Prof. MÜHL3eng in Aaran hat sich die Aufgabe gestellt, den östlichen Teil des Kettenjura im Massstabe 1:25000 aufzunehmen. Heute liegen uns bereits einige dieser mit grosser Mühe und Sorgfalt ausgearbeiteten Karten vor. Ganz besonderes Interesse ist auf die Gliederung und Darstellung der Diluvialablagerungen verwandt, die sich von jeher der Vorliebe MüHLBERG's erfreuten. Die beiden zuerst erschienenen Blätter umfassen die östlichen Ausläufer des Jura, die die Aare überschreiten. Der äusserste Sporn ist die Lägernkette, die sich in fast rein ostwestlicher Richtung von Baden an der Limmat bis Regensberg hinzieht. Diese Antiklinale zeigt schon sehr bald nach ihrem Auftauchen aus den Molasse- und Diluvialbedeckungen im Osten die Tendenz, sich nach Norden überzulegen. Der Nordschenkel wird steil gestellt und seine Schichten erfahren Reduktionen und Ausquetschungen. Zu bedeutenderen Überschiebungen kommt es östlich der Limmat noch nicht. Wir können MüHLBERG nicht beistimmen, wenn er dem Nordschenkel der Kette vorgelagerte, isoliert auf der Molasse liegende Malmkomplexe als durch Schub von Süden dorthin gebrachte Überschiebungsklippen deutet. Es dürfte sich vielmehr um Schollen handeln, die aus dem steilgestellten und mit Einsetzen der Erosion zum Teil überkippten Nordschenkel ausgebrochen und in ihre jetzige Lage abgerutscht sind. Muss MüHLBERg doch selbst zageben, dass der ans Muschelkalk gebildete Gewölbekern vollständig normal gebaut ist und keinerlei Störungen anfweist. Die Limmat benutzt za ihrem Durchbruch durch die Kette bei Baden eine Einsattelung der Scheitellinie und eine Transversalverschiebung im Nordschenkel der Kette. Weiter im Westen, zu beiden Seiten der Reuss, sehen wir den Muschelkalk des Gewölbekerns be- 
reits in mehreren Schuppen über den Nordschenkel der Antiklinale hinübergeschoben. Schon wegen des ganz analogen Baues möchten wir glauben, dass die Antiklinale der Habsburg an der Aare die westliche Fortsetzung der Kette bei Hausen und der Lägern überhaupt ist. MüHLbERG will dagegen das Habsburggewölbe nach Osten hin in einer kleinen Aufsattelung des Nordschenkels der Antiklinale bei Hausen ausklingen lassen. Leider ist der Zusammenhang der Kette hier durch ausgedehnte Diluvialablagerungen wiederholt verdeckt. Der Kestenberg im Süden der Habsburg ist das Ostende einer südlicheren Jurakette. Der Nordschenkel der Falte ist in die Tiefe gebrochen, die Stirn des hochgebliebenen Gewölbescheitels lokal über dessen steil in die Höhe geschleppten und überkippten Schichten vorgedrungen. Nördlich von den eben geschilderten Ketten liegt hier im Osten zunächst eine flache, von Molasse erfüllte Mulde, die Lengnaver Mulde, die von der Aare bei Brugg auf kürzere Erstreckung durchflossen wird. Nach Osten erweitert sich die Mulde, nach Westen spitzt sie sich za und hebt sich vollends aus, so dass der Tafeljura direkt an den Kettenjura stösst. Den Nordrand der Mulde bildet eine von Ober-Endingen nach Lauffohr verlaufende Flexur, in der sich die Juraschichten wieder herausheben und gegen Norden hin die flache Platte des Tafeljura bilden.

Die westlich an diese Blätter anschliessende Karțe der Umgebung von Aarau ist auch schon erschienen, sie umfasst nur ein Stück der südlichen Ketten, weshalb sie erst später besprochen werden soll. Inzwischen macht die geologische Aufnahme immer weitere Fortschritte. Grosse Strecken im Gebiete des Hauensteins, der Klusen von Önsingen und Mümliswil, sowie der Passwangkette sind in Ausarbeitung begriffen. Wichtige Fragen wie das Verhalten der Verwerfungen m Kettenjura zur Faltung, und die Beziehungen der Brüche des Tafeljura zum gefalteten Gebirge werden durch sie ihrer Lösung näher gebracht werden. Bald wird sich Gelegenheit bieten, über diese Untersuchungen hier wieder zusammenfassend zu berichten.

\section{Der Jura im Umkreis des lemurischen Kontinentes.}

Von E. Dacqué, Nünchen.

Mit 1 Kartenskizze.

Benuitzte Literatur.

1. Bozнм, G.: Reisenotizen aus Ostasien. Ztschr. deutsch. geol. Ges. Bd. 52. 1900. S. 554.

2. - - Reisenotizen aus Neuseeland. Ztschr. deutsch. geol. Ges. Bd. 52. 1900 S. 169 .

3. - - Aus den Molukken. Ztschr. deutsch. geol. Ges. Bd. 53. 1901. S. 8.

4. - - Neues aus d. Indo-Australischen Archipel. Beil.-Bd. XXII. z. N. Jahrb f. Min. etc. 1906. S. 385 .

5. - - Beiträge zur Geologie v.. Niederiänd.-Indien (Oxford d. Wai Galo) Palaeontographica. Suppl. IV. 1907. S. 120.

6. - - Jura v. Timor, Babas, Buru. Beil.-Bd. XXV z. N. Jahrb. f. Min. etc 1907. S. 324. 